\title{
Comparison of Markov versus quantum dynamical models of human decision making
}

\author{
Jerome R. Busemeyer* Peter D. Kvam† Timothy J. Pleskac ${ }^{\circledR}$
}

\author{
Article Type:
}

Advanced Review

\begin{abstract}
What kind of dynamic decision process do humans use to make decisions? In this article, two different types of processes are reviewed and compared: Markov and quantum. Markov processes are based on the idea that at any given point in time a decision maker has a definite and specific level of support for available choice alternatives, and the dynamic decision process is represented by a single trajectory that traces out a path across time. When a response is requested, a person's decision or judgment is generated from the current location along the trajectory. By contrast, quantum processes are founded on the idea that a person's state can be represented by a superposition over different degrees of support for available choice options, and that the dynamics of this state form a wave moving across levels of support over time. When a response is requested, a decision or judgment is constructed out of the superposition by 'actualizing' a specific degree or range of degrees of support to create a definite state. The purpose of this article is to introduce these two contrasting theories, review empirical studies comparing the two theories, and identify conditions that determine when each theory is more accurate and useful than the other.
\end{abstract}

*Department of Psychological and Brain Sciences, Indiana University

†Department of Psychology, University of Florida

${ }^{\ddagger}$ Department of Psychology, University of Kansas

§Please direct correspondence to Jerome Busemeyer jbusemey@indiana.edu, Peter Kvam pkvam@ufl.edu, or pleskac@ku.edu. All authors contributed equally to this paper. 


\section{GRAPHICAL TABLE OF CONTENTS}
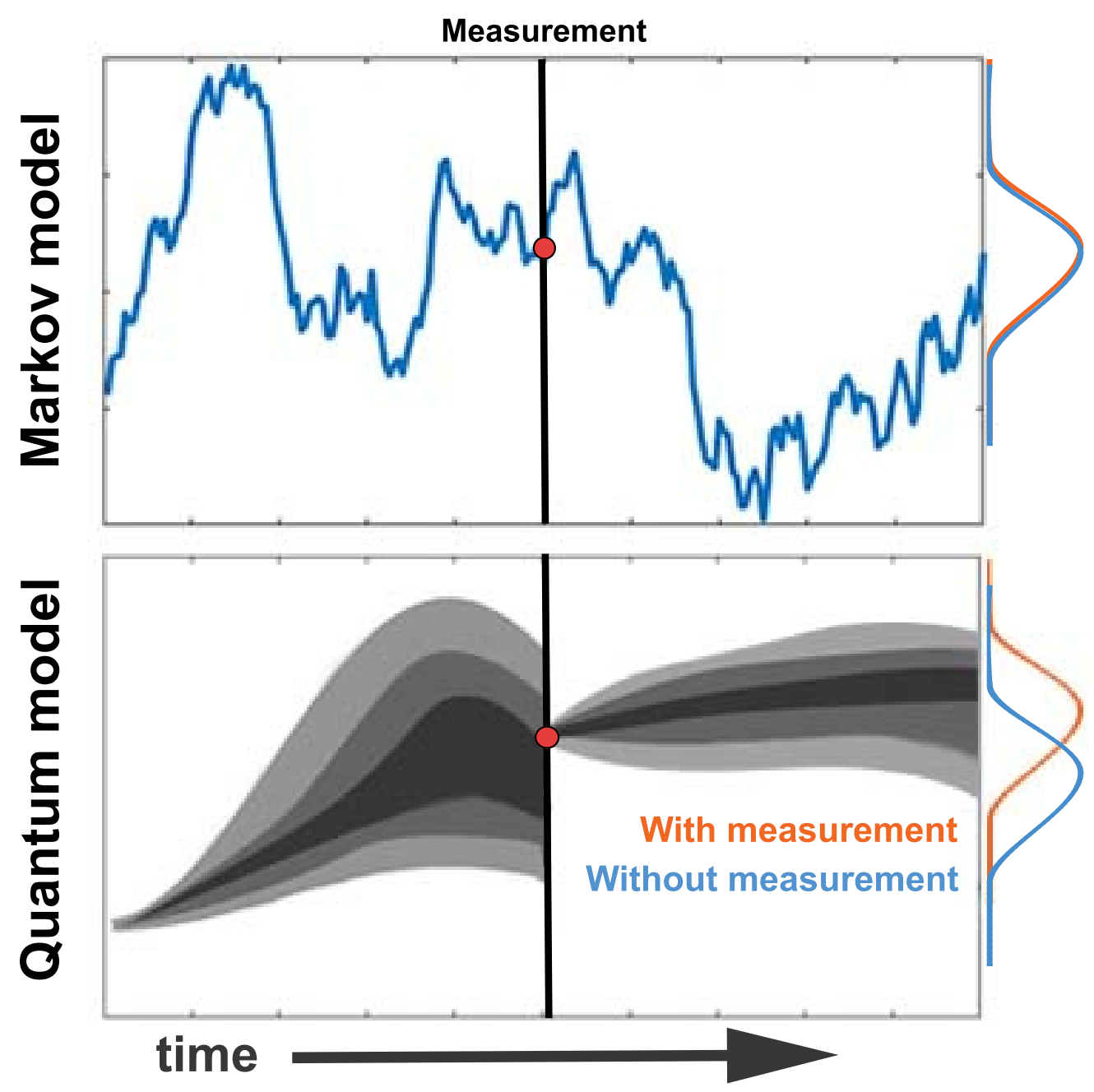

Figure 1: Markov and quantum random walk models generate diverging predictions for how evidence evolves over time and how measurements like decisions interact with subsequent responses.

\section{INTRODUCTION}

Imagine watching a murder mystery film with a friend. As you watch, you become aware of your beliefs about guilt or innocence of a suspect. Your beliefs move up and down across time as different kinds of evidence are presented during the movie scenes. At any point in 
time, your friend may ask which character seems the most guilty, or evaluate the evidence with respect to a particular character's guilt or innocence. When prompted, you can express the likelihood that the suspect is guilty or innocent to your friend.

Now imagine trying to decide whether or not to risk passing a car on a two lane highway. As you deliberate, you become aware of your affective evaluations toward taking the risk or not. Your tendency to approach or avoid changes across time as you monitor the driving situation. At each point in time you can decide to take the risk (pass) or not (stay).

These are two different kinds of decision problems. In the first case, the decision variable being monitored is the evidence for or against an hypothesis: an inferential choice problem. In the second case, the decision variable being monitored is your preference for or against an action: a preferential choice problem. Despite their differences, both decisions seem to be based on the same or similar process where evidence or preference is accumulated over time to eventually trigger a choice (Dutilh \& Rieskamp, 2016; Summerfield \& Tsetsos, 2012; Pleskac et al., 2019; Usher \& McClelland, 2004, 2001; Zeigenfuse et al., 2014). What are the basic dynamics that underlie the changes in these decision variables, evidence or preference, during decision making? And how are the judgments and actions about these decision variables generated from the latent monitoring and accumulation process? This article compares and contrasts two different ways to model the decision processes underlying these choices: a classical Markov process, and a non-classical quantum process. These two approaches to modeling dynamic decision behavior have been compared on a variety of tasks and measures, including sequential decisions (Busemeyer et al., 2009a; Wang \& Busemeyer, 2016b), decisions with subsequent confidence (Kvam et al., 2015) or preference ratings (Kvam, 2014; Wang \& Busemeyer, 2016a), sequences of judgments and ratings (Busemeyer, Kvam, \& Pleskac, 2019), and response time distributions (Busemeyer et al., 2006; Fuss \& Navarro, 2013). We begin by introducing the basic ideas underlying these two theories using the evidence accumulation problem. Later in the article we consider the preference accumulation problem. In the end, we introduce a more general "open system" quantum Markov approach that incorporates elements of both frameworks to provide a more complete description of how support for different choice options changes over time. 

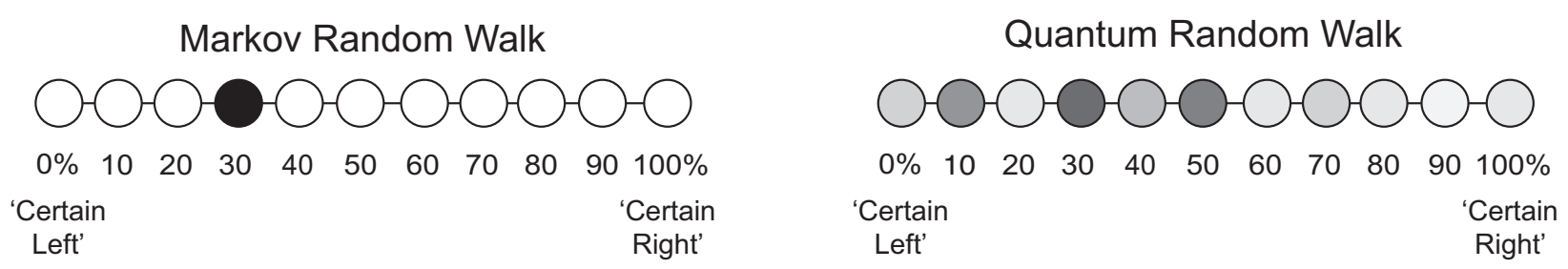

Figure 2: Diagram of a state representation of a Markov and a quantum random walk model. In the Markov model, evidence (shaded state) evolves over time by moving from state to state, occupying one definite evidence level at any given time. In the quantum model the decision-maker is in an indefinite evidence state, with each evidence level having a probability amplitude (shadings) at each point in time.

\section{Markov and quantum views of evidence accumulation}

The "classical" dynamical view of evidence accumulation, including the popular decision diffusion models (Ratcliff et al., 2016), asserts that a person's state of belief about a hypothesis at any single moment can be represented as a specific point along some internal scale of evidence, as illustrated in the left panel of Figure 2. This belief state changes moment by moment from one location to another on the evidence scale, carving out a trajectory as illustrated in the top panel of Figure 1. At the point in time when you are asked to report your belief, you simply read out the location on the evidence scale that existed before you were asked. That is, the report is determined by the pre-existing location of the belief state. This classical view of belief change is typically formalized as a Markov process (Bhattacharya \& Waymire, 1990), which describes a probability distribution over the evidence scale at each moment in time (shown in the left panel of Figure 3). This probability distribution represents a modeler's uncertainty about the location of the state that exists for a decision-maker at any point in time, rather than the decision maker's inherent uncertainty about the location of his or her own. A key assumption of Markov processes is that the probability distribution at the next moment in time only depends on the previous probability distribution and the dynamics of the state.

An alternative "non-classical" dynamical view of evidence accumulation asserts that your belief about a hypothesis at any single moment is not located at a specific point on the 
mental evidence scale. Instead, at any moment, it is superposed with some potential (called an amplitude) of realization across the scale, as illustrated by the shades of grey spread across the scale in right panel of Figure 2. As this superposition changes, it forms a wave that flows across levels of support over time. A representation of this wave, where darker regions correspond to greater squared probability amplitudes (corresponding to a greater likelihood of observing the state in that location), is shown in the bottom panel of Figure 1. When a person is asked to report their belief, a location of the evidence must be identified from this superposed state. This collapses the wave to a specific location or range of levels of support, depending on what type of measurement (binary choice, confidence judgment, or some other response) is applied. This "non-classical" view of belief change has been formalized as a quantum process (Gudder, 1979), which describes the amplitude distribution over the evidence scale across time as shown in the right panel of Figure 3 (but using squared amplitudes). Like the Markov process, it is based on the assumption that the amplitude distribution at the next moment in time only depends on the previous amplitude distribution.
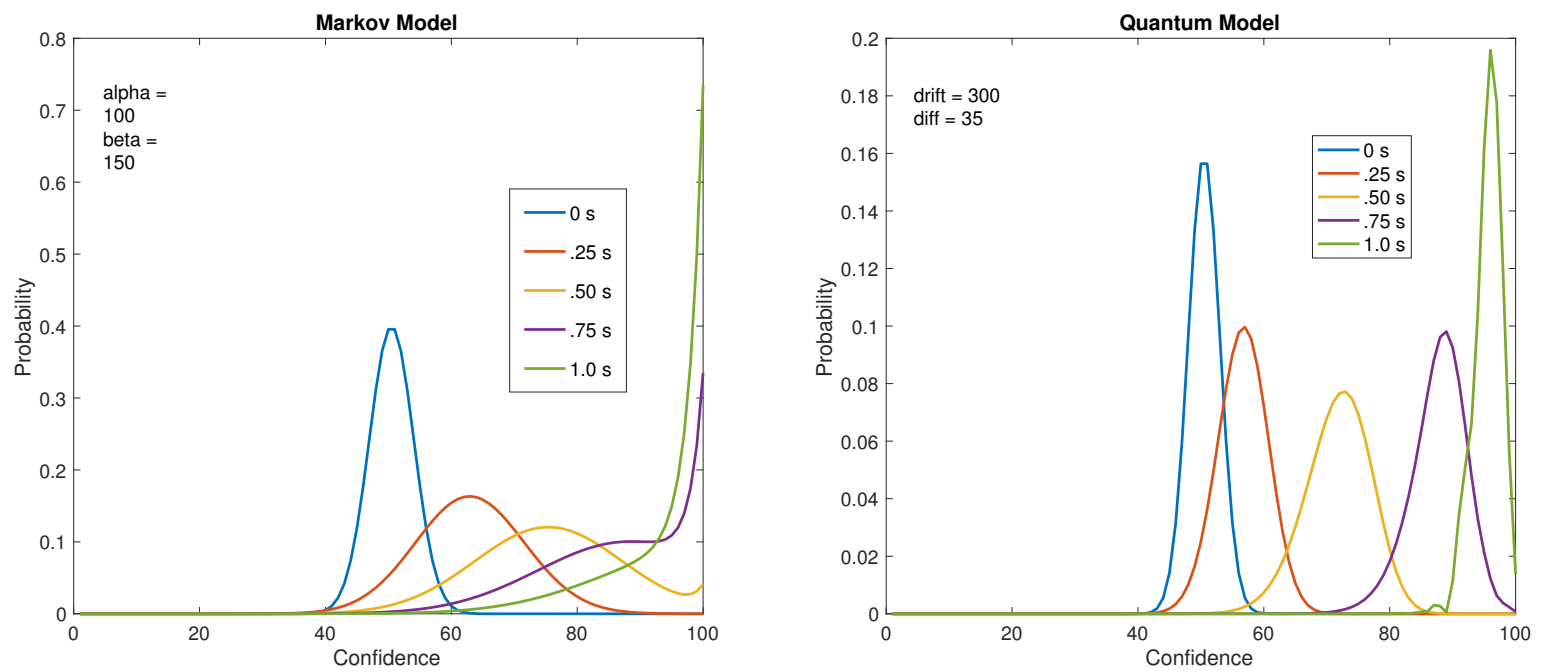

Figure 3: Illustration of Markov (Left) and quantum (Right) evolution of probability distributions over time. The horizontal axis represents 101 belief states associated with subjective evidence scale values ranging from 0 to 100 in one-unit steps. The vertical axis represents probability corresponding to each evidence level. The separate curves moving from left to right represent increasing processing time. 
Both Markov and quantum processes are stochastic processes. However, the probabilities used in Markov processes (Figure 3, left panel) and the squared amplitudes used in quantum processes (Figure 3, right panel) are conceptually quite different. On the one hand, the probabilities of Markov processes represent epistemic uncertainty, which is an observer's (e.g., a modeler's) lack of knowledge about the underlying true state existing at each moment in time due to a lack of information about the decision maker's beliefs. On the other hand, the amplitudes of quantum processes represent ontic uncertainty, which is the intrinsic uncertainty about the constructive result that a measurement generates at each moment in time (Atmanspacher, 2002). Ontic uncertainty cannot be reduced by greater knowledge about the decision maker's state.

These are two strikingly different views about the nature of change in belief during evidence accumulation. Markov processes, which include the popular random walk/diffusion models (Ratcliff et al., 2016; Diederich \& Busemeyer, 2003), are more established, and have a longer history with many successful applications. These include both the simple random walk models, where a decision maker has a discrete set of states (e.g., 11 confidence levels, 0/10/20/.../100) that they move through over time, shown in Figure 2, and the more common diffusion models where the "states" are a continuously-valued level of evidence (such as 0100, including all numbers in between). Since the discrete-state random walks approach a diffusion process as the number of states gets very large, we group these two approaches together under the umbrella of Markov process, which have been used to model choices and response times (Emerson, 1970; Luce, 1986; Stone, 1960) as well as probability judgments (Edwards et al., 1963; Wald \& Wolfowitz, 1949, 1948; Kvam \& Pleskac, 2016; Moran et al., 2015; Ratcliff \& Starns, 2009; Yu et al., 2015) in domains such as memory (Ratcliff, 1978), categorization (Nosofsky \& Palmeri, 1997), and inference (Pleskac \& Busemeyer, 2010).

Although quantum processes have a long and successful history in physics, they have only recently been considered for application to human decision making (Busemeyer \& Bruza, 2012; A. Y. Khrennikov, 2010). However, a series of studies have aimed at testing and comparing these two competing views of evidence accumulation. The purpose of this article is to (a) provide an introduction to these two contrasting views, (b) review the research that directly tests and compares these types of theories, and (c) draw conclusions about 
Table 1: Five basic principles underlying Markov and quantum dynamics

\begin{tabular}{lcc}
\hline \multicolumn{1}{c}{ Principle } & Markov & Quantum \\
\hline 1: State representation & Probability distribution & Amplitude distribution \\
2: State evolution & Transition operator & Unitary operator \\
3: Rate of change & Kolmogorov equation & Schrödinger eqaution \\
4: Dynamic operators & Intensity operator & Hamiltonian operator \\
5: Response Selection & Measurement operator & Measurement operator \\
\hline
\end{tabular}

conditions that determine when each view is more valid, or whether a mixture of the two processes might be needed for a more complete theory.

\section{Basic Principles of Markov and quantum dynamics}

Although the introduction focused on evidence accumulation that occurs during inferential choice problems, Markov and quantum models are based on general principles that can be applied to both evidence and preference accumulation problems. Table 1 provides a side by side comparison of the five general principles upon which the two theories are based. Both theories begin with a set of possible basic states that the system can pass through over time, describing the relative degrees of support for one option or the other. In the case of evidence accumulation, these states are distinct levels of belief. Figure 2 uses 11 levels, but actual applications typically use a much larger number so as to approximate a continuum. In the case of preference accumulation, these states are distinct levels of preference.

State representation principle For the Markov model, there is a probability distribution across states at each point in time. The probability assigned to each basic state conceptually represents the likelihood that an outside observer might attribute to the decision maker being located at that state. This probability distribution always sums to one. For the quantum model, there is an amplitude distribution across states at each point in time. The amplitude assigned to a basic state can be a real or complex number, and the probability of reporting that state is the squared magnitude of the 
amplitude. ${ }^{1}$ The sum of squared amplitudes always sums to one (i.e., the amplitude distribution has unit length). The nonlinear map from amplitudes to probabilities is an important way the two theories differ, and as we will see in the next section on an interference effect, it leads to testable competing predictions.

State evolution principle For the Markov model, the probability distribution over states evolves for period of time $t>0$ according to a linear transition operator. This operator describes the probability of transiting from one basic state to another over some period of time $t$, representing how incoming information changes the probability distribution over states across time. For the quantum model, the amplitude distribution evolves for a period of time $t>0$ according to a linear unitary operator. This operator describes the amplitude for transiting from one basic state to another over some period of time $t$. Once again, the probability of making this transition is obtained from the squared magnitude. In other applications, the unitary operator can also specify how the state changes according to some fixed amount of new information, such as a single vignette (Trueblood \& Busemeyer, 2011) or cue in the choice environment (Busemeyer et al., 2011). The unitary operator is required to maintain a unit length amplitude distribution over states across time, thus constraining the sum of squared amplitudes representing the probability of observing different measurement outcome to be equal to one.

Rate of change principle For the Markov model, the rate of change in the probability distribution is determined by a linear differential equation called the Kolmogorov equation. The integration of these momentary changes are required to form a transition operator. For the quantum model, the rate of change in the amplitude distribution is determined by the Schrödinger equation. The integration of these momentary changes are required to form a unitary operator. These two linear differential equations are not shown here, but they are strikingly similar, except for the complex number $i$ that ap-

\footnotetext{
${ }^{1}$ The use of complex numbers is common in mathematics including signal processing, time series analysis, and control theory. Complex numbers are used in quantum theory essentially as a mathematically efficient representation for computation of the probability amplitudes.
} 
pears in the Schrödinger equation (which is the reason for using complex amplitudes).

Dynamic Operators For the Markov model, the linear differential equation is defined by an intensity operator that contains two key parameters: a "drift rate" parameter that determines the direction of change and a "diffusion" parameter that determines the dispersion of the probability distributions. The intensity operator of the Markov model has to satisfy certain properties to guarantee that the linear differential equation produces a transition operator. For the quantum model, the linear differential equation is defined by a Hamiltonian operator that also contains two key parameters: a parameter that determines the "potential function" which controls the direction of change and a "diffusion" parameter that determines the dispersion of the amplitude distribution. The Hamiltonian operator of the quantum model has to satisfy different properties than the intensity operator to guarantee that the linear differential equation produces a unitary operator, but it serves a similar function by evolving the corresponding state according to incoming information. These operators are the key ingredients of any stochastic processing theory and they contain the most important parameters for building a model.

Response selection For the Markov model, the probability of reporting a response at some point in time equals the sum of the probabilities over the states that map into that response. After observing a response, a new probability distribution, conditioned on the observed response, is formed for future evolution. This probability distribution represents the updated information of an outside observer, where the measurement has reduced their uncertainty about the decision-maker's state. For the quantum model, the probability of reporting a response at some point in time equals the sum of the squared amplitudes over the states that map into that response. After observing a response, a new amplitude distribution, conditioned on the observed response, is formed for future evolution. In quantum models, this conditioning on the observed response is sometimes called the "collapse" of the wave function, which represents a reduction in the ontic uncertainty about the decision maker's evidence level, informing both the decision maker and an outside observer about the decision maker's cognitive 
state.

Despite the apparent similarities between the quantum and Markov processes outlined in the table, the two processes produce quite distinct behavior. For example, consider two processes shown in Figure 3 again. The Markov process in this figure is analogous to a pile of sand with wind blowing the sand to the right, where the sand eventually piles up in an equilibrium distribution. The quantum process in this figure is more closely analogous to a wave of water with the wind blowing the wave to the right. Once the wave hits the right wall, it bounces back until the wind blows it forward again. The result is that the quantum model does not reach an equilibrium, and instead it oscillates back and forth to the right across time. This interesting behavior is examined later in this article when we discuss preferential evolution. The following sections review previous applications of these models to evidence accumulation problems like choice, confidence, and response time, followed by applications to preference evolution.

\section{Interference effects}

The state representation and the response selection process in the Markov process relies on a property that we call the read-out assumption. In Markov models, a judgment or a decision is made by mapping an existing state of evidence onto a response. For instance, a choice is made when evidence reaches a predetermined level of evidence, triggering the appropriate response. Other responses are modeled similarly as a read-out from an existing level of evidence: confidence, for instance, is typically modeled by mapping predetermined levels of evidence to confidence ratings (Moran et al., 2015; Pleskac \& Busemeyer, 2010; Ratcliff \& Starns, 2009), as are preference ratings (Bhatia \& Pleskac, 2019) and judgments like willingness to pay, willingness to accept, and certainty equivalent prices (Johnson \& Busemeyer, 2005; Kvam \& Busemeyer, 2019).

This read-out assumption bears a striking resemblance to the assumption in economic models that preferences and beliefs are revealed by the choices people make (McFadden et al., 1999; Samuelson, 1938). Yet decades of research from judgment and decision making and behavioral economics suggests that preferences are not revealed by the choices people make, 
but rather constructed by the process of generating a response like a choice (Ariely \& Norton, 2008; Lichtenstein \& Slovic, 2006; Payne et al., 1992; Slovic, 1995). This construction of preference is typically understood as the result of people selecting a specific procedure from a larger repertoire of possible strategies to formulate a response (Gigerenzer et al., 1999; Hertwig et al., 2019; Payne et al., 1993; Tversky et al., 1988), or the dynamic nature of information accumulation that adjusts preferences over time (Busemeyer \& Townsend, 1993; Johnson \& Busemeyer, 2005). But quantum models, which model a judgment or decision as a measurement process that creates or constructs a definite state from an indefinite (superposition) state, offer a potentially more apt account of this hypothesis that preferences and beliefs are constructed.

\section{Interference effects of choice on confidence}

What are the behavioral implications of this process of constructing a definite state from an indefinite state? If information processing stops after the choice or judgment, then behaviorally this process is hard to dissociate from the classical read-out assumption of Markov models. However, if processing continues after a choice is made, then the two theories can make very different predictions about subsequent judgments. For instance, consider the situation when people are asked to make a choice and then rate their confidence in their choice - such paradigms are common in studies designed to study metacognition and confidence (Lichtenstein et al., 1982; Yeung \& Summerfield, 2012). If we model evidence accumulation during this situation as a Markov process, then a choice is a read-out of the location of the evidence, and the subsequent confidence judgment is just another read-out. However, we also know that in general evidence accumulation does not stop after the choice and that people continue to collect evidence after their choice (Baranski \& Petrusic, 1998; Moran et al., 2015; Pleskac \& Busemeyer, 2010; Yu et al., 2015). Consequently, a confidence judgment is made based on the evidence accumulated to make a choice as well as the evidence from postdecisional processing. Because classical models of evidence accumulation do not change the state of the evidence when it is measured, making a choice does not have an impact on the confidence people report. Thus, if a choice is made, but we ignore the choice, then the confidence should be the same as if they made no choice at all. In comparison, in 


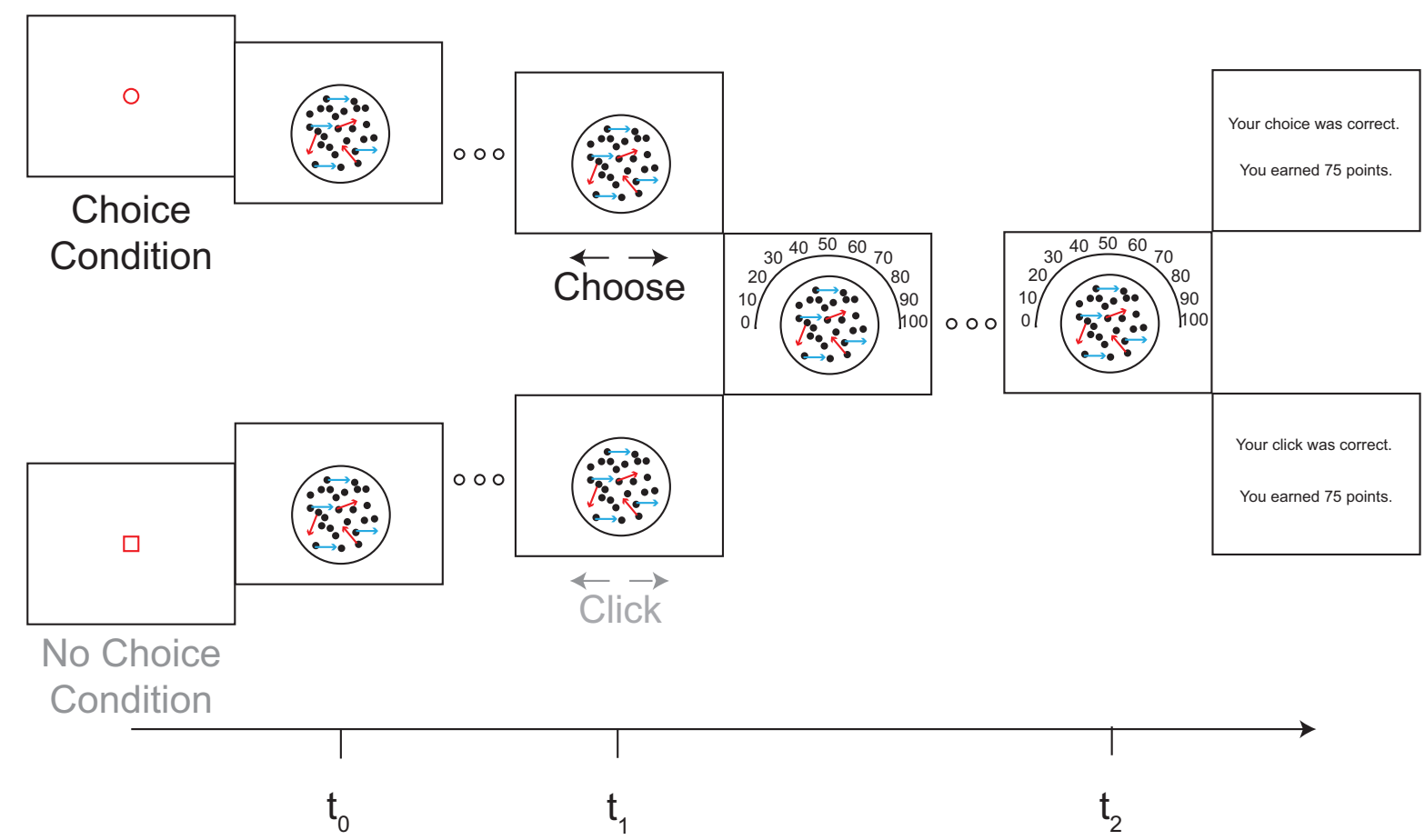

Figure 4: Diagram of the choice-confidence task. A fixation point indicated the choice/nochoice condition, then the stimulus was shown for $0.5 \mathrm{~s}$. A prompt $\left(t_{1}\right)$ then cued a decision on the direction of the dot motion (choice condition) or a motor response (no-choice condition). The stimulus remained on the screen. A second prompt $\left(t_{2}\right)$ then cued a confidence rating on the direction of the dot motion. Finally, feedback was given on the accuracy of their responses.

a quantum process, a choice does changes the state of the evidence when a choice is made, and so even if we ignore the choice, the simple act of measurement does impact confidence judgments following subsequent evidence accumulation.

These are parameter-free competing predictions that hold for a large range of evidence accumulation processes, including ones where there is decay and trial-by-trial variability (for a mathematical proof, see Kvam et al., 2015). In general, they arise from the first-principles of each of the theories. To get an intuitive feel for the predictions, consider the experiment shown in Figure 4 that Kvam et al. (2015) asked participants to complete. In the experiment, participants viewed a random dot motion stimulus where a percentage of the dots moved coherently in one direction (left or right), and the rest moved randomly. In half of the blocks 
of trials participants were prompted at time point $t_{1}(0.5 \mathrm{~s})$ to decide whether the coherently moving dots were moving left or right and entered their choice via a mouse. In the the other half of the blocks - the no-choice condition-participants were prompted at $t_{1}$ to click a pre-determined mouse button. In all the trials, at a second time point $t_{2}$, participants were prompted to rate their confidence that the coherently moving dots were moving right ranging from 0 (certain left) to $100 \%$ (certain right).

The prediction of no interference from the Markov model arises because it models evidence accumulation as the evolution of the probability distribution over evidence levels across time. In the choice condition, according to the state evolution principle in Table 1, the probability distribution evolves during the first time interval. Next, according to the response selection principle, the probability of a particular choice is the probability of the evidence summing across the evidence levels associated with that response. Then after a choice, the evidence continues to accumulate, but now the observer has less (epistemic) uncertainty about the location of the evidence (i.e., at time $t_{1}$ we know the accumulated evidence was located within the levels associated with the choice that was reported) so the probability distribution evolves until the end of the second time interval conditional on the choice at time $t_{1}$. It is at this second time point $t_{2}$ that this conditional probability distribution is used to compute the probability of a confidence rating. In the no-choice condition, the probability distribution over the evidence levels continues to evolve up until time point $t_{2}$ and is used to compute the probability of a particular confidence rating. Critically, the probability distribution over evidence levels at $t_{2}$ in the no-choice condition is identical to the marginal distribution over evidence levels at the same point in time in the choice condition. This is because the Markov process obeys the Chapman - Kolmogorov equation, which is a dynamic form of law of total probability (Bhattacharya \& Waymire, 1990). Thus, at $t_{2}$ the probability of a confidence judgment in the no-choice condition is identical to the total probability of a confidence judgment in the choice condition across the two different possible choices (correct or incorrect) at $t_{1}$,

$$
\begin{aligned}
\operatorname{Pr}\left(\text { conf }=y \mid t_{2}, \text { no-choice }\right)= & \operatorname{Pr}\left(\text { correct } \cap \text { conf }=y \mid t_{2}, \text { choice }\right)+ \\
& \operatorname{Pr}\left(\text { incorrect } \cap \text { conf }=y \mid t_{2}, \text { choice }\right) .
\end{aligned}
$$

In the quantum model, according to the state representation and evolution principles, ev- 
idence accumulation is an evolution of an amplitude distribution over evidence levels across time. In the choice condition, the amplitude distribution describing the indefinite state of evidence evolves during the first time interval until the time $t_{1}$. According to the response selection principle of the quantum model, the probability of a choice is computed from the sum of the squared magnitudes of the amplitudes associated with that response, and thereafter, the indefinite state collapses to a state definitely located among the states associated with the choice. After the choice, the amplitude distribution then evolves from the definite state to a new indefinite state before the second confidence rating. At the second time point $t_{2}$, the probability of the confidence judgment is determined from the probability amplitudes associated with that judgment. In the no-choice condition, the probability amplitudes evolve until $t_{2}$, at which point the probability of a particular confidence judgment is the sum of the squared amplitudes associated with that judgment. However, unlike the Markov model, the marginal probability distribution over the different evidence levels (and thus confidence) at time $t_{2}$ in the choice condition is not the same as the probability distribution over the different evidence levels (and thus confidence) at time $t_{2}$ in the no-choice condition,

$$
\begin{array}{r}
\operatorname{Pr}\left(\text { conf }=y \mid t_{2}, \text { no-choice }\right) \neq \operatorname{Pr}\left(\text { correct } \cap \text { conf }=y \mid t_{2}, \text { choice }\right)+ \\
\operatorname{Pr}\left(\text { incorrect } \cap \text { conf }=y \mid t_{2}, \text { choice }\right) .
\end{array}
$$

The difference arises because the square of the sum of probability amplitudes from the nochoice condition is not the same as the sum of the squared amplitudes from the choice condition.

Kvam et al. (2015) ran the experiment shown in Figure 4 and found that, on average, decisions during the task interfered with subsequent confidence judgments, resulting in less extreme and more accurate judgments than when no decision was elicited. With the large number of trials collected, the predictions were also tested at the individual level. In this case, six of the nine participants showed a credible interference effect. The three participants who did not show the effect were in fact still consistent with the prediction of the quantum model, as they did not show credible evidence for continuing to accumulate evidence after making a choice; this second-stage processing is a necessary condition for observing the interference effect. As a further comparison between the two processes, a Bayesian model 
comparison was conducted between the quantum random walk model and several different Markov random walk models. Based on the Bayes factors, across all nine participants the quantum random walk model provided a better account of the data than any of the Markov models, including Markov models that were adjusted to give rise to an interference effect.

\section{Interference effects of confidence on confidence}

The process of constructing a response from an indefinite state is not just limited to choices, but potentially extends to all responses. It stands to reason then that the interference effects we observed in Kvam et al. should also occur with other responses. To test this, Busemeyer, Kvam, \& Pleskac (2019) ran a similar experiment asking participants to make two probability judgments. ${ }^{2}$ This study used a similar procedure as the procedure of the choice-confidence study, with participants judging the direction of motion of a random dot motion stimulus that (between trials) had different amounts of dots coherently moving together $(2 \%, 4 \%, 8 \%$, and $16 \%$ ). Participants were asked to make two probability ratings one at time point $t_{1}$ and the other at time point $t_{2}$ (see Figure 5 ). The experiment included three main conditions: (1) requests for probability ratings at times $t_{1}=.5 \mathrm{~s}$ and $t_{2}=1.5 \mathrm{~s},(2)$ requests for ratings at times $t_{1}=1.5 \mathrm{~s}$ and $t_{2}=2.5 \mathrm{~s}$, and (3) requests for ratings at times $t_{1}=.5 \mathrm{~s}$ and $t_{2}=2.5 \mathrm{~s}$.

Using this design, interference effects were assessed by comparing the marginal distribution of probability ratings at time $t_{2}=1.5 \mathrm{~s}$ for condition 1 (pooled across ratings made at time $t_{1}=.5 \mathrm{~s}$ ) with the distribution of ratings at time $t_{1}=1.5 \mathrm{~s}$ from condition 2 . As before, the Markov model predicted no difference between conditions at the matching time points, whereas the quantum model predicted an interference effect of the first rating on the second. The effects were more subtle - the results produced significant differences only for the lowest coherence levels, and were present at the individual level for only 3 out of the 11 participants at the low $(2 \%, 4 \%)$ coherence levels. Thus, the the results suggest that

\footnotetext{
${ }^{2}$ The study in Busemeyer, Kvam, \& Pleskac (2019) was originally designed to test the temporal Bell inequality described later in this article by examining reversals in confidence (see also Box 1). However, the results were inconclusive particularly because the temporal Bell inequality was designed for choice instead of confidence.
} 


\section{Condition 1: Model Calibration}

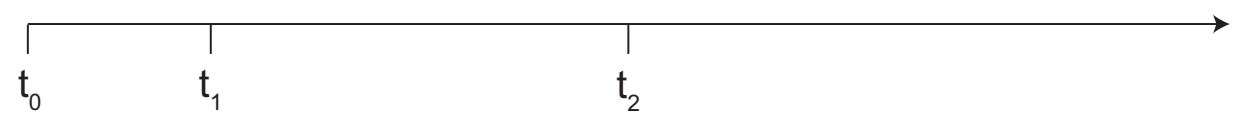

\section{Condition 2: Model Calibration}

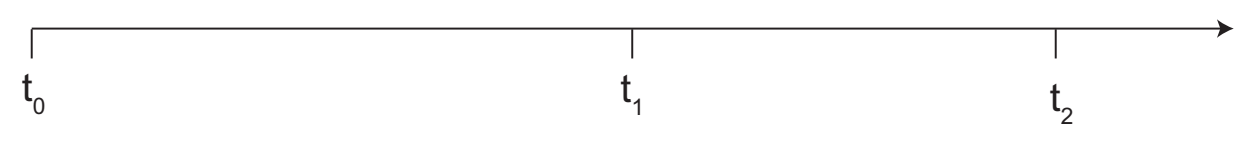

Condition 3: Generalization

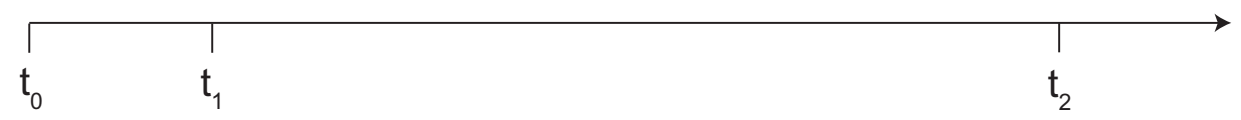

Figure 5: Illustrations of design for double confidence judgment experiment. Each condition had a different pair of time points $\left(t_{1}\right.$ and $\left.t_{2}\right)$ for requests for ratings: the time intervals of the first two conditions are contained within condition 3. Conditions 1 and 2 were used to estimate model parameters and then these same model parameters were used to predict the ratings for condition 3 . The time point $t_{0}$ corresponds to stimulus onset.

interference effects do occur with sequences of judgments, but they are small and occur for only a subset of the participants and coherence conditions. One way to interpret this difference in empirical results is that using a binary decision for the first measurement may be more effective for producing a "collapse" as compared to making a probabilistic judgment, potentially suggesting that different types of measurements may have different effects on the evidence state.

This experiment also made it possible for a generalization test (Busemeyer \& Wang, 2000) to quantitatively compare the Markov and quantum models. Unlike the Bayes factor method previously used by Kvam et al. (2015), the generalization test provides a method to test a priori predictions of the models in new conditions. To do this, the parameters of the models were estimated using results obtained from conditions 1 and 2 for each individual; and then these same parameters were used to predict probability ratings for each person on the third condition (see Figure 5). For 8 of the 11 participants the comparison favored the quantum model for coherence levels $2 \%, 4 \%$, and $8 \%$, but only 5 participants produced 
results favoring the quantum model for coherence level $16 \%$. The results clearly favored the quantum model overall, but less so for high coherence. These results indicated that some features of both Markov and quantum models may be needed to accurately account for the results. The quantum model seems to perform better at the low coherence levels and the Markov model begins to do better at the higher coherence levels.

\section{Categorization-decision paradigm}

Of course, choice and confidence judgments about dot motion direction are not the only situations in which people may be tasked with making multiple responses in sequence. Another illustration of the effects of sequential responses comes from a paradigm where participants were asked to make a category judgment about a face shown on the screen and a decision about how to interact with that face (Townsend et al., 2000). In this study, the faces shown on screen could belong to one of two groups: a hostile group and a friendly group. The categorization judgment, when prompted, asked participants to determine which group the face belonged based on the relative width of the face (for example, wider faces were more likely to be friendly and narrower faces more likely to be hostile). The decision component of the task regarded how to interact with that face: to act defensively or to act friendly. The optimal behavior was to act defensively in response to hostile / narrow faces, and act friendly with friendly / wide faces.

The key manipulation in this study was whether or not the defensive/friendly decision was preceded by a categorization judgment, or whether the decision was made alone (and similarly, whether the categorization judgment would be affected if it were preceded by an action decision). It was designed to test the Markov assumption that the marginal probabilities of acting defensive/friendly should not depend on whether or not there was a categorization judgment preceding it. Although the time course of these responses was not precisely controlled as in the interference studies above, the result similarly violated the law of total probability. Participants were more likely to act defensively when the decision was presented alone (without the categorization beforehand) than when it was preceded by the additional response (Busemeyer et al., 2009a; Wang \& Busemeyer, 2016b). Conversely, Busemeyer et al. (2009b) developed a quantum model that was permitted to violate the law of total 
probability, but constrained to obey another law called double stochasticity. Participants' decisions more closely followed the predictions of the quantum model by violating the law of total probability without violating the law of double stochasticity. This constitutes another domain in which interference between a sequence of responses generates a pattern of results in direct conflict with predictions of a Markov model.

Similar quantum models have been used to explain findings such as the disjunction effect in the Prisoner's dilemma (Tversky \& Shafir, 1992; Pothos \& Busemeyer, 2009) and two-stage gambling paradigms (Shafir \& Tversky, 1992; Busemeyer et al., 2015), and other measurement effects on preference (Sharot et al., 2010; White et al., 2014, 2016). However, these experiments do not examine the time at which responses are made and the corresponding models tend to have simpler non-dynamical structures, so they are not reviewed here.

\section{Summary}

Taken together, these experiments help us hone in on the conditions in which the evidence accumulation may best be described as a quantum process and why. First, as we have established, based on first-principles, the Markov models predict that earlier responses do not impact the evidence accumulation process that helps determine later responses. Yet all three experiments show an interference effect such that earlier responses impact later ones. Generally speaking, the interference effect appears to be stronger when the first response is a binary one such as choice. One way to interpret this difference in empirical results is that using a binary decision for the first measurement may be more effective for producing a "collapse" as compared to making a probabilistic judgment. However, it is not just the interference effect that is consistent with a quantum process. For instance, in Kvam et al. the quantum model even provided a better fit than Markov models that we modified to recreate the interference effect. Part of the reason is that any modifications to the Markov model to account for the interference effect are post-hoc and add additional complexity to the model, whereas the quantum model predicts the interference effect from its first principles. Finally, the observed confidence distributions are frequently multi-modal and discontinuous. The Markov model again does not account for these properties with its first principles. However, 
the study by Busemeyer, Kvam, \& Pleskac shows that this comparative advantage is more evident at lower levels of coherence, suggesting that the accumulation process may take a more classical form when selections are easier-possibly due to most of the probability amplitude being unaffected when a decision is easy (most of the amplitude will favor the correct decision, and so the collapse at $t_{1}$ removing amplitude below $50 \%$ will have little effect on the state of evidence).

So far the key property that we have used to distinguish Markov versus quantum dynamics in this section is interference of a first response on later responses. However, other qualitative properties can also be tested in future work. One important property in particular is called the temporal Bell inequality (see Box 1 Atmanspacher \& Filk, 2010). This is a test concerning an inequality based on comparing binary decisions at three different time intervals. Markov models must satisfy this inequality and quantum models can violate this property. In fact, Atmanspacher et al. (2004) proposed a quantum model of bistable perception that violates the temporal Bell and provided some preliminary evidence that supports this prediction. Yearsley \& Pothos (2014) laid out specific tests of these inequalities and the classical notion of cognitive realism, and outlined judgment phenomena constituting violations of these inequalities that could be accounted for by quantum, but not classical, models of cognition. ${ }^{3}$

Choice and confidence are two of the three most important and widely studied measures of cognitive performance (Vickers \& Packer, 1982; Vickers, 2001). The third measure is response time. Arguably, it is the ability of random walk/diffusion models to account for response times that has made them so popular (Ratcliff et al., 2016). Next we explore to what degree a quantum account may provide a new perspective on response times as well.

\footnotetext{
${ }^{3}$ Cognitive realisim is the idea that a definite cognitive state is always present at any definite point in time.
} 


\section{Box 1}

The temporal bell inequality was introduced to cognitive science by Atmanspacher and Filk 2010 to test their dynamic quantum model of bistable perception. In particular, they were investigating the rate of change in perceived orientation of a Necker cube. However, the test can be applied to any sequence of binary decisions that satisfy the design shown in Figure 5 (see also Footnote 2). In the case of the Necker cube, participants would be asked a binary question such as "does the cube appear orientated up or down." Denote $D$ as the event of changing the perceived orientation from one time point to another time point (either changing from up to down or changing from down to up), and then define $p\left(D \mid t_{a}, t_{b}\right)$ as the probability of changing orientation from the time point $t_{a}$ to another time point $t_{b}$. Then, referring to Figure 5 , the temporal Bell inequality is expressed as $p\left(D \mid t_{1}, t_{2}\right)+p\left(D \mid t_{2}, t_{3}\right) \geq$ $p\left(D \mid t_{1}, t_{3}\right)$. All Markov models must satisfy this inequality, assuming as usual, that the particular measurement pair does not change the dynamics of the system (the system can be non-stationary, but it is assumed to be non-stationary in the same way for all pairs). The reason why is because the Markov model implies that all three of these probabilities of change can be derived from a single common three way joint distribution of the 2 (state is up or down at time $\left.t_{1}\right) \times 2$ (state is up or down at time $\left.t_{2}\right) \times 2$ (state is up or down at time $t_{3}$ ), and this three way joint distribution must satisfy the temporal Bell inequality. If the inequality is violated, then no 3-way joint distribution even exists. Atmanspacher and Filk showed that their quantum dynamic model for the Necker cube paradigm can indeed violate this inequality for some specially selected time points.

\section{Response time Experiments}

One of the most important and well-studied measures of cognitive processes is that of response times (Luce, 1986). Although judgments at different time points provide an important window onto the cognitive processes underlying the representation and revision of evidence, the ones we have discussed thus far have been responses elicited at specific experimentally controlled time points. A natural step forward is to predict characteristics of selections 
made in optional stopping procedures, where the decision maker, rather than experimenter, is in control of when a decision or judgment is made. In a typical choice-response time experiment, the decision maker is presented with a noisy stimulus (like the dot motion task stimulus shown in Figure 4), and views the stimulus until the decision maker decides when to stop and make a choice. Across many trials of this kind of experiment, a researcher can collect a distribution of choices and response times to each stimulus condition. The models are then designed to predict the choice proportions (usually accuracy in terms of percent correct) and response time distributions.

The optional stopping procedure raises an interesting issue concerning the stopping rule. The usual assumption for making at choice at a particular time for the Markov model is to stop as soon as the process reaches a criterion level. For example, referring to Figure 2, the criterion for stopping and choosing "certain right" might be when the process first reaches the $90 \%$ confidence level. This stopping rule is made possible by the assumption that the Markov process is located at a specific level at each time point. Unlike the Markov process, the quantum process is not located at any specific level of evidence at each point in time. Referring again to Figure 2, the quantum walk process is superposed over levels until a measurement is taken. Therefore, one must make an additional assumption about when the state is observed, such as supposing that the decision maker repeats measurements at regular small time intervals. Each measurement results in one of three categorizations: one that produces a result that reaches or exceeds the upper criterion (e.g., > 90\%); one that produces a result that reaches or falls below the lower criterion (e.g., $<10 \%$ ); or a measurement that produces a result falling in between the two criteria. If the measurement produces a result that exceeds a criterion, then the process stops and that measurement is reported. If the measurement produces a result in between the criterion, then the process continues for another step. The final decision time is the sum of the time units for each repeated measurement until the process stops, producing a discrete approximation of response time distributions commonly used in decision models (Ratcliff et al., 2016).

Figure 6 gives a general idea about the predictions for response time from each type of model. The parameters of the two models were chosen to give approximately the same probability of correct. There are several features to note. First, the quantum model has a faster 

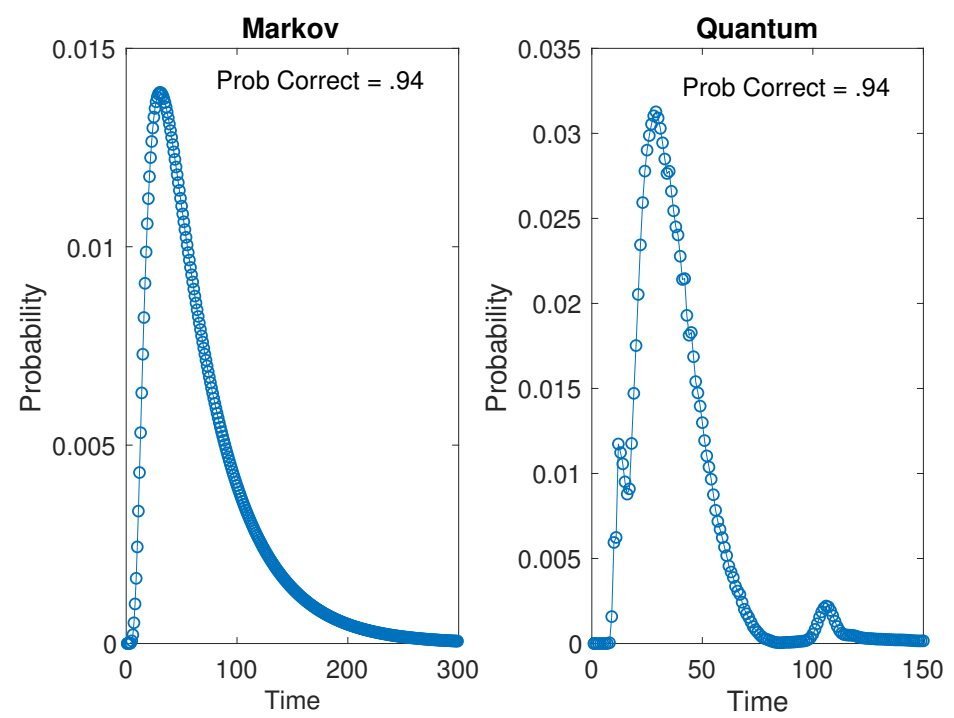

Figure 6: Comparison of stopping times for Markov (left panel) and quantum (right panel) models. Horizontal axis is time (in the same arbitrary units for both models) and vertical axis is relative frequency of a stopping time. The parameters were set so that both models produce the same choice accuracy. Note that the horizontal and vertical scales are different because the quantum model is faster than the Markov model.

stopping time as compared to the Markov model in this example. This property has also been observed by other researchers (Fuss \& Navarro, 2013), and may constitute an adaptive reason for why a decision maker would implement a quantum rather than Markov random walk for decision making. Second, the quantum model has a small second mode, which is produced by oscillation and interference properties of the quantum dynamics. Empirically multi-modal distributions have been interpreted as evidence for multiple stage processes (see for example Pleskac \& Wershbale, 2014); however, in this case there is only a single process. Furthermore, empirical distributions are often smoothed, which could make it difficult to detect a small second mode in the distribution.

The first comparison between models with regard to response times was carried out by Busemeyer et al. (2006). In that work, the distributions of response times were derived from quantum and Markov models by assuming that a choice was made as soon as the state was measured at a location beyond a specific criterion level. Measurements were assumed to occur every $10 \mathrm{~ms}$ to determine if the process had crossed the choice boundary by that time. 
This initial effort at comparing models in terms of response time predictions favored the Markov model, which was nearly equivalent to the well established diffusion decision model of response times (Ratcliff et al., 2016). Although this first comparison did not favor the quantum model, it did show that the quantum model was capable of providing reasonably accurate fits to the choice and response time distributions.

This early work comparing quantum and Markov models of response time was then followed up by subsequent developments by Fuss \& Navarro (2013), who used a more general approach to modeling quantum dynamical systems. The previous work by Busemeyer et al. (2006) was limited by its use of what is called a "closed" quantum system that does not include any additional "noise" operators and always remains in a coherent (superposed) state. Fuss \& Navarro (2013) implemented a quantum random walk that included quantum "noise" operators that generate partial decoherence, producing a decay into a part-quantum and part-classical system that mixed both von Neumann uncertainty (measurement uncertainty about where a superposition state will collapse) and classical uncertainty (uncertainty about which of several states a person is located). This type of 'open' quantum system represents a potentially more realistic view of quantum random walk models (Yearsley, 2017), where the superposition state partially decoheres as a result of interactions with "noise" (as opposed to the "pure" coherent states presented above that are part of a closed quantum system without any "noise"). This partially-coherent quantum model can be interpreted as a massively parallel cognitive architecture that involves both inhibitory and excitatory interactions between units (e.g., neurons or neural populations), as we might expect from neural representations of evidence. It turns out that this more general quantum walk model outperformed a simple diffusion model in fitting the response time distributions in a perceptual decision-making experiment (Gökaydin et al., 2011).

With results running in both directions between Markov and quantum models, it is too early to say which type of model is more promising for modeling response times. Quantitative tests based on model fits may not produce a clear answer for distinguishing these two type of response time models. Instead, the quality of out-of-sample predictions like the generalization test (similar to that presented in the interference with double confidence ratings section) may be necessary to arbitrate between them. Nevertheless, the open quantum systems approach, 
inspired by the cooperative and competitive interactions between units representing evidence (neurons), appears a promising direction for developing better models of response times.

\section{Preference and dissonance}

As mentioned at the beginning, Markov and quantum process are also applicable to understanding how preferences accumulate and evolve over time. A great deal of work has already been done applying Markov models to preference (Busemeyer, Gluth, et al., 2019; Pleskac et al., 2015), but we are only beginning to apply quantum processes to preference evolution. Two initial applications are described below.

The quantum model is equally applicable whether the underlying scale is degrees of preference or degrees of belief, and so it makes extremely similar predictions regarding the effect of a binary choice on subsequent preference ratings as it does with confidence ratings. Namely, it suggests that preference ratings that follow a choice, when there is information processing between the choice and preference rating, should diverge from those that are not preceded by a choice as in the interference from choice on confidence study above. The effect of a decision on subsequent preferences that arises from the quantum walk models bears some interesting commonalities with other well-studied phenomena. In particular, the observation that making a decision results in different distributions of confidence judgments is reminiscent of work on cognitive dissonance that was applied to preference judgments (Festinger, 1957, 1964). In this work, a decision maker is offered a choice between two alternatives, and then is subsequently (after some delay following choice) asked to rate their preference between the choice options. The typical finding is that post-choice preference ratings favor the chosen alternative, relative to either pre-choice preferences (Brehm, 1956) or to a preference elicited in absence of prior choice (Festinger \& Walster, 1964).

The typical explanation for dissonance effects is one of motivated reasoning: A person is driven by conflict between internal states of preference (A and B are similar in value) and stated preference elicited via choice (A chosen over B) to change their degree of preference to favor the chosen option. However, this 'bolstering' effect is sometimes preceded by an opposing 'suppression' effect, where a chosen alternative is more weakly preferred to 
an alternative compared to cases where there is no decision between between the options (Festinger \& Walster, 1964; Walster, 1964; Brehm \& Wicklund, 1970). Both of these effects are clearly at odds with a Markov account of preference representation, which suggests that making a decision by itself should not change alter an underlying preference state between a pair of options.

Conversely, the quantum dynamical models provide a natural explanation for these bolstering and suppression effects. Although the quantum account is not incompatible with the dissonance account of bolstering based on motivational processes, the quantum framework offers an alternative explanation for these effects. For example, White et al $(2014 ; 2016)$ used measurement effects with a (non-dynamic) quantum model to account for decision biases that unfolded in sequential affective judgments.

Here, we take this work a step further by using a dynamical model to account for preferences measured at experimentally at different controlled points in time. According to quantum dynamics, the measurement of a choice at an early time point creates a cognitive state that interacts with subsequent accumulation dynamics. Therefore a quantum process predicts that a choice at an early time point naturally results in subsequent preferences that diverge from those produced by a no-choice condition. As with the choice-confidence interference study, we expect a paradigm eliciting choice and then preference to yield an interference effect where preference ratings in a no-choice condition systematically differed from those in a choice condition.

\section{Oscillation}

One implication of the quantum approach to preference formation and dissonance is that it predicts bolstering and suppression effects should depend on the time at which preference ratings are elicited. The choice-confidence interference study used a relatively short timescale between choice and confidence ratings (maximum 1.5 seconds after choice), and generated an effect closer to suppression, where ratings were more extreme in the no-choice than in the choice condition. However, due to the oscillatory nature of quantum models, we also expect to find the reverse phenomenon of bolstering (choice $>$ no-choice) when confidence or preference strength is elicited at a later point in time. 

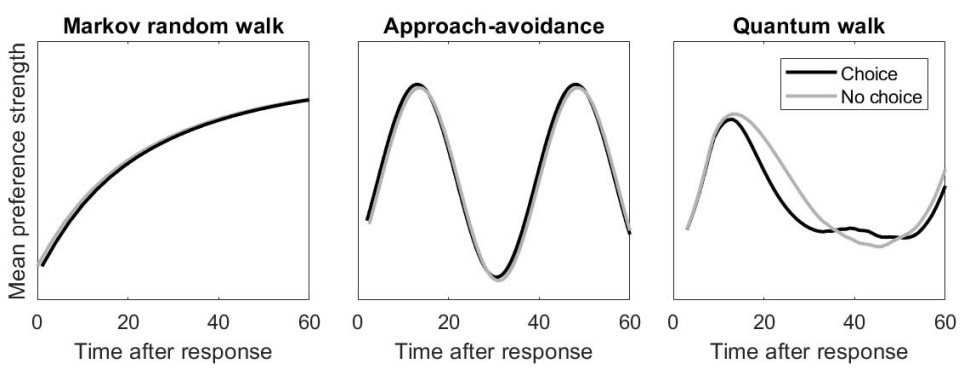

Figure 7: Expected time course of mean preference ratings generated from a typical Markov random walk model (left), a deterministic oscillating approach-avoidance model (middle), and quantum walk model (right).

An example of model predictions for how preference should evolve over time for a Markov, a determistic oscillator, and a quantum model are shown in Figure 7. As shown, the quantum model predicts that preference strength should oscillate over time, owing to the wave-like dynamics described in Table 1. Choice dampens the magnitude of these oscillations, leading to instances of both suppression (no-choice $>$ choice, around 5-35 seconds after choice in Figure 7) and bolstering (choice $>$ no-choice, around 25-50 seconds after choice in Figure 7). Naturally, the time at which each type of effect appears will depend on the stimuli used as choice alternatives, the individual characteristics of the decision-maker, and thus the corresponding parameters of the model (such as drift rate and diffusion). Work exploring this unique oscillation prediction from the quantum model is still underway, but early suggestions are that oscillations do indeed appear in preference data when ratings are elicited at varying time points, and that the resulting preference pattern exhibits both bolstering and suppression effects in line with the quantum model predictions and in contrast to the Markov model (Kvam, 2014).

The results of the first experiment exploring the time course of mean preference strength are shown in Figure 8 (this is a re-analysis of the data from Kvam, 2014). In this experiment, participants completed a choice condition, where they chose between two gift cards after 5 seconds and then rated their degree of preference between them at 3, 6, 8, 18, 30, or 45 seconds after choice; or a no-choice condition, where the choice response was replaced with a button press, as in the interference study presented above. As predicted by the quantum model, preference strength shifted back and forth over time, creating a pattern that exhibits 


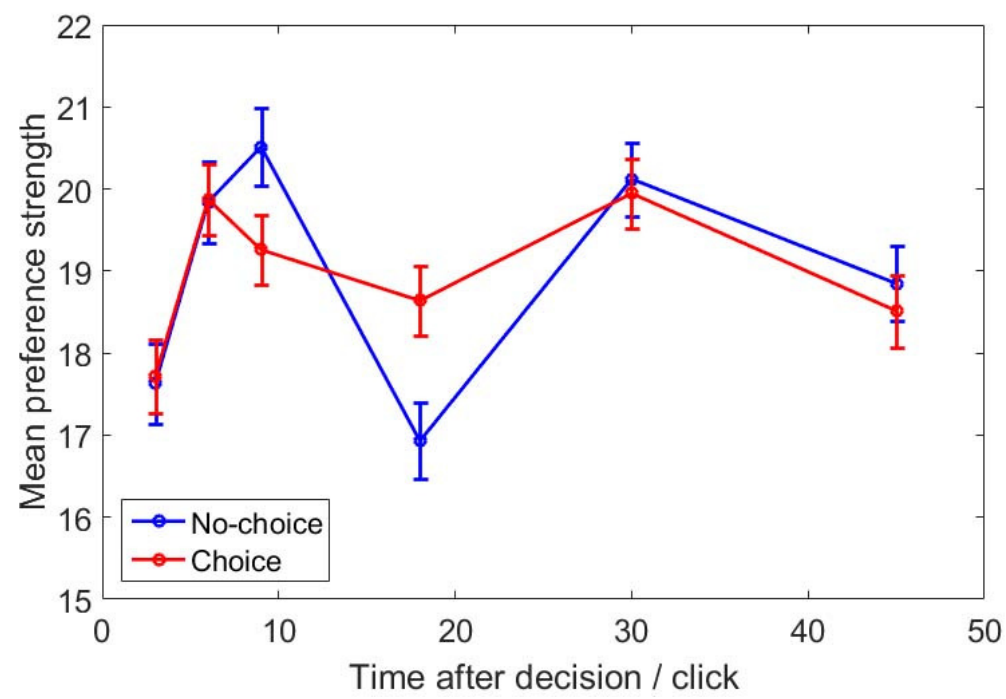

Figure 8: Pattern of mean preference strength elicited at different time points following a decision (choice) or irrelevant button press (no-choice). Error bars indicate \pm 1 unit of standard error - differences between choice and no-choice are substantive at 9- and 18-second time points.

some oscillations. Furthermore, preference strength shifts are dampened (stabilized) by a prior choice, resulting in a difference in mean preference between choice and no-choice at different time points in different directions. Because the pattern of choice and no-choice reverses due to the oscillations, there are both early suppression effects (no-choice > choice, around 9s following initial response) as well as the classic bolstering effect that has appeared in experiments investigating cognitive dissonance (choice $>$ no-choice, around 18s following initial response).

This pattern of observed mean preference strength is striking, showing a pattern of oscillation that is at odds with both our intuitions about preference as well as predictions of many classical models. In particular, it represents an experimental result that conflicts on multiple levels with a Markov account of preference, which suggests that choice and no-choice should not differ and that mean preference strength should increase monotonically with time. Although these early results are suggestive of a qualitative gap in performance between the two models, a formal model comparison should further reveal the degree to which the quantum and Markov models are capable of handling these patterns in the data. 


\section{Conclusion}

The comparison between Markov and quantum models of evidence accumulation is particularly illuminating because subtle but systematic differences in how they represent evidence states, measurement, and evidence accumulation lead to diverging predictions regarding the effects of cognitive measurements like decisions or confidence judgments. These a priori predictions offer the rare opportunity to discriminate between models on the basis of qualitative results - such as the interference effect - rather than only based on quantitative model comparison.

Foremost among these diverging predictions is the idea from the quantum model that decision making is a constructive process, where taking a cognitive measurement by making a choice creates rather than simply records the state of a cognitive system. This aligns with the long-standing but mathematically informal theory in the decision-making literature that choice is a constructive process that requires a person to generate a new cognitive state in order to respond in decision scenarios (Ariely \& Norton, 2008; Lichtenstein \& Slovic, 2006; Payne et al., 1992; Slovic, 1995). The effect of this measurement process is illustrated in experiments like the choice-confidence paradigm, which resulted in distributions of confidence that depended on whether or not choice was elicited at an earlier time point, referred to here as an interference effect (Kvam et al., 2015). Related work investigating the effect of categorization on subsequent decisions in the categorization-decision paradigm (Townsend et

al., 2000; Busemeyer et al., 2009a) again illustrates the effect of measuring cognition through categorization decisions and its effect on subsequent decisions about how to interact with other agents.

Although both of these paradigms illustrate gaps in the Markov approach to modeling decisions, other results seem more mixed regarding which type of model is most appropriate. The high coherence conditions of the double confidence experiment revealed that the Markov model can out-perform the quantum model when there is not clear interference between sequential responses (Busemeyer, Kvam, \& Pleskac, 2019). Other types of data like response times, where Markov models have long been applied and vetted, also serve as challenges where Markov models out-perform quantum models (Busemeyer et al., 2006). With such a 
long history of application to modeling response times (Ratcliff et al., 2016), it is perhaps not too surprising that this measure is more within the purview of classical Markov and diffusion models. Further development and testing of the nascent dynamic quantum models may prove that both types of models are competitive in these scenarios, but for now it seems that the classical approach can yield quantitatively superior fits.

One concern regarding the differences between Markov and quantum models of evidence accumulation is the plausibility in terms of implementations of these systems. While a description of behavior in quantum terms does not necessitate a corresponding neurophysiological implementation, it would certainly be more convincing if this connection could be made. The Markov model has natural connections to neural substrates through classical neural networks (Kira et al., 2015; Rao, 2010), making it straightforward to imagine it being implemented in the brain. It is potentially possible that quantum operations are implemented via true superposition (pure quantum states) in the brain (Hagan et al., 2002), although this would have to occur such a noisy environment that quantum states are likely to decohere immediately (Tegmark, 2000). A potentially more likely explanation is that a classical neural network produces patterns that are described well by the mathematics of quantum theory. Exactly this kind of classical neural network implementation of quantum computations also have been proposed (Busemeyer et al., 2017; A. Khrennikov et al., 2018), providing a simple but plausible connection between quantum walks and neural substrates. Likewise, an "open system" quantum model possesses many properties that naturally align with classical neural systems, including parallel processing along with excitatory and inhibitory interactions between adjacent states (neural populations) (Fuss \& Navarro, 2013).

This article has focused on contrasting quantum and Markov models. However, really both frameworks have insights to offer regarding the evidence accumulation and decision process. Indeed, these models co-exist within "open" quantum systems, which begin with ontic uncertainty of the quantum model (where the decision maker is uncertain about their own state due to superposition over evidence levels) and later reduce to epistemic uncertainty (where the decision maker knows their state, but the modeler does not due to the existence of probabilistic dynamics) through interaction with the noise in the internal or external environment. This "decoherence" takes us from a pure "closed" quantum system described 
by superposition to a partially-coherent or mixed system described by a combination of both superposition and classical uncertainty. Such a system can be described in terms of density matrices, quantum "noise" operators, and mixed states characterizing both our uncertainty about the state a decision maker is in as well as the decision maker's uncertainty about how their state might be mapped onto to possible responses or levels of evidence. Ultimately, this may provide a more comprehensive account: while quantum models are necessary to account for effects like interference, there is undoubtedly a degree of internal (metacognitive) measurement and classical uncertainty about what state a person is located that should be incorporated into the model, creating a mix of quantum and classical uncertainty. Such a mix is often left unexamined in more traditional accounts of decision making under uncertainty (see for example Hertwig et al., 2019). These types of hybrid quantum and Markov models for decision making are developed in work by several researchers (Yearsley, 2017; Fuss \& Navarro, 2013; Asano et al., 2011; Khrennikova, 2016). Moving forward, these "open system" models show great promise and may even be necessary to account for the multifaceted results on evidence accumulation, decision-making, preference, and confidence.

\section{References}

Ariely, D., \& Norton, M. I. (2008). How actions create - not just reveal - preferences. Trends in Cognitive Sciences, 12(1), 13-16. doi: 10.1016/j.tics.2007.10.008

Asano, M., Ohya, M., Tanaka, Y., Basieva, I., \& Khrennikov, A. (2011). Quantum-like model of brain's functioning: Decision making from decoherence. Journal of Theoretical Biology, 281(1), 56-64.

Atmanspacher, H. (2002). Determinism is ontic, determinability is epistemic. In H. Atmanspacher \& R. Bishoph (Eds.), Between chance and choice: interdisciplinary perspectives on determinism (pp. 49-74). Thorverton, UK: Imprint Academic.

Atmanspacher, H., \& Filk, T. (2010). A proposed test of temporal nonlocality in bistable perception. Journal of Mathematical Psychology, 54, 314-321. 
Atmanspacher, H., Filk, T., \& Romer, H. (2004). Quantum zero features of bistable perception. Biological Cybernetics, 90, 33-40.

Baranski, J. V., \& Petrusic, W. M. (1998). Probing the locus of confidence judgments: experiments on the time to determine confidence. Journal of Experimental Psychology: Human Perception and Performance, 24 (3), 929-945.

Bhatia, S., \& Pleskac, T. J. (2019). Preference accumulation as a process model of desirability ratings. Cognitive Psychology, 109, 47-67.

Bhattacharya, R. N., \& Waymire, E. C. (1990). Stochastic processes with applications. Wiley.

Brehm, J. W. (1956). Postdecision changes in the desirability of alternatives. The Journal of Abnormal and Social Psychology, 52(3), 384-389. doi: 10.1037/h0041006

Brehm, J. W., \& Wicklund, R. A. (1970). Regret and dissonance reduction as a function of postdecision salience of dissonant information. Journal of Personality and Social Psychology, 14(1), 1-7. doi: 10.1037/h0028616

Busemeyer, J. R., \& Bruza, P. D. (2012). Quantum models of cognition and decision. Cambirdge University Press.

Busemeyer, J. R., Fakhari, P., \& Kvam, P. (2017). Neural implementation of operations used in quantum cognition. Progress in Biophysics and Molecular Biology, 130, 53-60.

Busemeyer, J. R., Gluth, S., Rieskamp, J., \& Turner, B. M. (2019). Cognitive and neural bases of multi-attribute, multi-alternative, value-based decisions. Trends in Cognitive Sciences.

Busemeyer, J. R., Kvam, P., \& Pleskac, T. J. (2019). Markov versus quantum dynamic models of belief change during evidence monitoring. Scientific Reports, 9(1), 1-10.

Busemeyer, J. R., Pothos, E. M., Franco, R., \& Trueblood, J. S. (2011). A quantum theoretical explanation for probability judgment errors. Psychological Review, 118 (2), 193-218. 
Busemeyer, J. R., \& Townsend, J. T. (1993). Decision field theory: A dynamic-cognitive approach to decision making in an uncertain environment. Psychological Review, $100(3)$, 432-459. doi: 10.1037//0033-295X.100.3.432

Busemeyer, J. R., \& Wang, Y.-M. (2000). Model comparisons and model selections based on generalization criterion methodology. Journal of Mathematical Psychology, 44(1), 171189.

Busemeyer, J. R., Wang, Z., \& Lambert-Mogiliansky, A. (2009a). Empirical comparison of markov and quantum models of decision making. Journal of Mathematical Psychology, 53(5), 423-433.

Busemeyer, J. R., Wang, Z., \& Lambert-Mogiliansky, A. (2009b). Empirical comparison of markov and quantum models of decision making. Journal of Mathematical Psychology, 53 (5), 423-433.

Busemeyer, J. R., Wang, Z., \& Shiffrin, R. S. (2015). Bayesian model comparison favors quantum over standard decision theory account of dynamic inconsistency. decision, 2, 1-12. Decision, 2, 1-12.

Busemeyer, J. R., Wang, Z., \& Townsend, J. T. (2006). Quantum dynamics of human decision-making. Journal of Mathematical Psychology, 50(3), 220-241.

Diederich, A., \& Busemeyer, J. R. (2003). Simple matrix methods for analyzing diffusion models of choice probability, choice response time, and simple response time. Journal of Mathematical Psychology, 47(3), 304-322.

Dutilh, G., \& Rieskamp, J. (2016). Comparing perceptual and preferential decision making. Psychonomic Bulletin \&3 Review, 23(3), 723-737. doi: 10.3758/s13423-015-0941-1

Edwards, W., Lindman, H., \& Savage, L. J. (1963). Bayesian statistical inference for psychological research. Psychological review, 70(3), 193.

Emerson, P. L. (1970). Simple reaction time with markovian evolution of gaussian discriminal processes. Psychometrika, 35(1), 99-109. 
Festinger, L. (1957). A theory of cognitive dissonance. Stanford, CA: Stanford University Press.

Festinger, L. (1964). Conflict, decision, and dissonance. Redwood City, CA: Stanford University Press.

Festinger, L., \& Walster, E. (1964). Post-decision regret and decision reversal. In L. Festinger (Ed.), Conflict, decision, and dissonance (pp. 100-112). Stanford, CA: Stanford University Press.

Fuss, I. G., \& Navarro, D. J. (2013). Open parallel cooperative and competitive decision processes: A potential provenance for quantum probability decision models. Topics in Cognitive Science, 5(4), 818-843.

Gigerenzer, G., Todd, P., \& the ABC Research Group. (1999). Simple heuristics that make us smart. New York, NY: Oxford University Press.

Gökaydin, D., Ma-Wyatt, A., Navarro, D., \& Perfors, A. (2011). Humans use different statistics for sequence analysis depending on the task. In Proceedings of the 33rd annual meeting of the cognitive science society (pp. 543-548). Retrieved from https://escholarship.org/uc/item/4hg0g273

Gudder, S. P. (1979). Stochastic methods in quantum mechanics. Dover.

Hagan, S., Hameroff, S. R., \& Tuszyński, J. A. (2002). Quantum computation in brain microtubules: Decoherence and biological feasibility. Physical Review E, 65(6), 061901.

Hertwig, R., Pleskac, T. J., Pachur, T., \& The Center for Adaptive Rationality. (2019). Taming uncertainty. MIT Press.

Johnson, J. G., \& Busemeyer, J. R. (2005). A dynamic, stochastic, computational model of preference reversal phenomena. Psychological Review, 112(4), 841-861. doi: 10.1037/ 0033-295X.112.4.841 
Khrennikov, A., Basieva, I., Pothos, E. M., \& Yamato, I. (2018). Quantum probability in decision making from quantum information representation of neuronal states. Scientific reports, 8(1), 16225 .

Khrennikov, A. Y. (2010). Ubiquitous quantum structure: From psychology to finance. Springer.

Khrennikova, P. (2016). Quantum dynamical modeling of competition and cooperation between political parties: the coalition and non-coalition equilibrium model. Journal of Mathematical Psychology, 71, 39-50.

Kira, S., Yang, T., \& Shadlen, M. N. (2015). A neural implementation of walds sequential probability ratio test. Neuron, 85(4), 861-873.

Kvam, P. D. (2014). The time course of preference formation: Quantitative process model predictions for cognitive dissonance effects (Unpublished master's thesis). Michigan State University.

Kvam, P. D., \& Busemeyer, J. R. (2019). A distributional and dynamic theory of pricing and preference. Preprint. doi: 10.31234/osf.io/3sx8b

Kvam, P. D., \& Pleskac, T. J. (2016). Strength and weight: The determinants of choice and confidence. Cognition, 152, 170-180. doi: 10.1016/j.cognition.2016.04.008

Kvam, P. D., Pleskac, T. J., Yu, S., \& Busemeyer, J. R. (2015). Interference effects of choice on confidence. , 112 (34). Proceedings of the National Academy of Science, 112 (34), 10645-10650.

Lichtenstein, S., Fischhoff, B., \& Phillips, L. D. (1982). Calibration of probabilities: The state of the art to 1980. In P. S. D. Kahneman \& A. Tversky (Eds.), .

Lichtenstein, S., \& Slovic, P. (2006). The construction of preference. Cambridge, UK: Cambridge University Press.

Luce, R. D. (1986). Response times: Their role in inferring elementary mental organization (No. 8). Oxford University Press on Demand. 
McFadden, D., Machina, M. J., \& Baron, J. (1999). Rationality for economists? In B. Fischhoff \& C. F. Manski (Eds.), Elicitation of preferences (pp. 73-110). Dordrecht: Springer. doi: 10.1007/978-94-017-1406-8-4

Moran, R., Teodorescu, A. R., \& Usher, M. (2015). Post choice information integration as a causal determinant of confidence: Novel data and a computational account. Cognitive Psychology, 78, 99-147. doi: 10.1016/j.cogpsych.2015.01.002

Nosofsky, R. M., \& Palmeri, T. J. (1997). An exemplar-based random walk model of speeded classification. Psychological Review, 104(2), 266-300. doi: 10.1037/0033-295X.104.2.266

Payne, J. W., Bettman, J. R., \& Johnson, E. J. (1992). Behavioral decision research: A constructive processing perspective. Annual Review of Psychology, 43, 87-131. doi: 10.1146/annurev.ps.43.020192.000511

Payne, J. W., Bettman, J. R., \& Johnson, E. J. (1993). The adaptive decision maker. New York, NY: Cambridge University Press.

Pleskac, T. J., \& Busemeyer, J. R. (2010). Two-stage dynamic signal detection: A theory of choice, decision time, and confidence. Psychological Review, 117(3), 864-901. doi: $10.1037 / \mathrm{A} 0019737$

Pleskac, T. J., Diederich, A., \& Wallsten, T. S. (2015). Models of decision making under risk and uncertainty. In J. Busemeyer, Z. Wang, J. T. Townsend, \& A. Eidels (Eds.), The oxford handbook of computational and mathematical psychology (pp. 209-231). New York, NY: Oxford University Press.

Pleskac, T. J., \& Wershbale, A. (2014). Making assessments while taking repeated risks: A pattern of multiple response pathways. Journal of Experimental Psychology: General, 143(1), 142-162. doi: 10.1037/a0031106

Pleskac, T. J., Yu, S., Hopwood, C., \& Liu, T. (2019). Mechanisms of deliberation during preferential choice: Perspectives from computational modeling and individual differences. Decision, 6(1), 77-107. doi: 10.1037/dec0000092 
Pothos, E. M., \& Busemeyer, J. R. (2009). A quantum probability model explanation for violations of 'rational' decision making. Proceedings of the Royal Society, B., 276 (1665), 2171-2178.

Rao, R. P. (2010). Decision making under uncertainty: a neural model based on partially observable markov decision processes. Frontiers in Computational Neuroscience, 4, 146.

Ratcliff, R. (1978). A theory of memory retrieval. Psychological Review, 85(2), 59-108. doi: $10.1037 / 0033-295 X .85 .2 .59$

Ratcliff, R., Smith, P. L., Brown, S. L., \& McCoon, G. (2016). Diffusion decision model: Current history and issues. Trends in Cognitive Science, 20, 260-281.

Ratcliff, R., \& Starns, J. J. (2009). Modeling confidence and response time in recognition memory. Psychological Review, 116(1), 59-83. doi: 10.1037/a0014086

Samuelson, P. A. (1938). A note on the pure theory of consumer's behaviour. Economica, $5(17), 61-71$.

Shafir, E., \& Tversky, A. (1992). Thinking through uncertainty: nonconsequential reasoning and choice. Cognitive Psychology, 24, 449-474.

Sharot, T., Velasquez, C. M., \& Dolan, R. J. (2010). Do decisions shape preference? evidence from blind choice. Psychological Science, 21 (9), 1231-1235.

Slovic, P. (1995). The construction of preference. American Psychologist, 50 (5), 364-371. doi: 10.1037/0003-066X.50.5.364

Stone, M. (1960). Models for choice-reaction time. Psychometrika, 25(3), 251-260.

Summerfield, C., \& Tsetsos, K. (2012). Building bridges between perceptual and economic decision-making: neural and computational mechanisms. Frontiers in neuroscience, 6, 70. doi: $10.3389 /$ fnins.2012.00070

Tegmark, M. (2000). Importance of quantum decoherence in brain processes. Physical review $E, 61(4), 4194$. 
Townsend, J. T., Silva, K. M., Spencer-Smith, J., \& Wenger, M. J. (2000). Exploring the relations between categorization and decision making with regard to realistic face stimuli. Pragmatics 85 Cognition, 8(1), 83-105.

Trueblood, J. S., \& Busemeyer, J. R. (2011). A quantum probability account of order effects in inference. Cognitive science, 35(8), 1518-1552.

Tversky, A., Sattath, S., \& Slovic, P. (1988). Contingent weighting in judgment and choice. Psychological Review, 95(3), 371-384. doi: 10.1037//0033-295X.95.3.371

Tversky, A., \& Shafir, E. (1992). The disjunction effect in choice under uncertainty. Psychological Science, 3, 305-309.

Usher, M., \& McClelland, J. L. (2001). The time course of perceptual choice: the leaky, competing accumulator model. Psychological Review, 108(3), 550-592. doi: 10.1037/ 0033-295X.108.3.550

Usher, M., \& McClelland, J. L. (2004). Loss aversion and inhibition in dynamical models of multialternative choice. Psychological Review, 111(3), 757-769. doi: 10.1037/0033-295X .111 .3 .757

Vickers, D. (2001). Where does the balance of evidence lie with respect to confidence? [Book Section]. In E. Sommerfeld, R. Kompass, \& T. Lachmann (Eds.), Proceedings of the seventeenth annual meeting of the international society for psychophysics (pp. 148-153). Lengerich: Pabst.

Vickers, D., \& Packer, J. (1982). Effects of alternating set for speed or accuracy on response time, accuracy and confidence in a unidimensional discrimination task. Acta Psychologica, 50(2), 179-197. doi: 10.1016/0001-6918(82)90006-3

Wald, A., \& Wolfowitz, J. (1948). Optimum character of the sequential probability ratio test. The Annals of Mathematical Statistics, 19(3), 326-339.

Wald, A., \& Wolfowitz, J. (1949). Bayes solutions of sequential decision problems. Proceedings of the National Academy of Sciences of the United States of America, 35(2), 99-102. 
Walster, E. (1964). The temporal sequence of post-decision processes. In L. Festinger (Ed.), Conflict, decision, and dissonance (pp. 112-128). Stanford, CA: Stanford University Press.

Wang, Z., \& Busemeyer, J. (2016a). Comparing quantum versus markov random walk models of judgements measured by rating scales. Philosophical Transactions of the Royal Society A: Mathematical, Physical and Engineering Sciences, 374(2058), 20150098.

Wang, Z., \& Busemeyer, J. R. (2016b). Interference effecs of categorization on decision making. Cognition, 150, 133-149.

White, L. C., Barqué-Duran, A., \& Pothos, E. M. (2016). An investigation of a quantum probability model for the constructive effect of affective evaluation. Philosophical Transactions of the Royal Society A: Mathematical, Physical and Engineering Sciences, $374(2058), 20150142$.

White, L. C., Pothos, E. M., \& Busemeyer, J. R. (2014). Sometimes it does hurt to ask: the constructive role of articulating impressions. Cognition, 133(1), 48-64.

Yearsley, J. M. (2017). Advanced tools and concepts for quantum cognition: A tutorial. Journal of Mathematical Psychology, 78, 24-39.

Yearsley, J. M., \& Pothos, E. M. (2014). Challenging the classical notion of time in cognition: A quantum perspective. Proceedings of the Royal Society B: Biological Sciences, $281(1781), 20133056$.

Yeung, N., \& Summerfield, C. (2012). Metacognition in human decision-making: confidence and error monitoring. Philosophical Transactions of the Royal Society B: Biological Sciences, 367(1594), 1310-1321.

Yu, S., Pleskac, T. J., \& Zeigenfuse, M. D. (2015). Dynamics of postdecisional processing of confidence. Journal of Experimental Psychology: General, 144(2), 489-510. doi: 10.1037/ xge0000062 
Zeigenfuse, M. D., Pleskac, T. J., \& Liu, T. (2014). Rapid decisions from experience. Cognition, 131(2), 181-194.

\section{FURTHER READING}

Bruza, P. D., Wang, Z., \& Busemeyer, J. R. (2015) Quantum cognition: A new theoretical approach to psychology. Trends in Cognitive Science, 19 (7), 383-393.

Busemeyer, J. R., \& Wang, Z. (2015). What is quantum cognition and how is it applied to psychology? Current Directions in Psychological Science, 24 (3), 163-169

Pothos, E. M., \& Busemeyer, J. R. (2013). Can quantum probability provide a new direction for cognitive modeling? Behavioral and Brain Sciences, 36, 255-274. (Target Article).

Kvam, P. D., \& Pleskac, T. J. (2017). A quantum information architecture for cue-based heuristics. Decision, 4, 197-233.

Wang, Z., Busemeyer, J. R., Atmanspacher, H., \& Pothos, E. M. (2013). The potential of using quantum theory to build models of cognition. Topics in Cognitive Science, 5(4), $672-688$. 